\title{
Collocation Method for Multiplicative Noise Removal Model
}

\author{
Mushtaq Ahmad Khan ${ }^{1 a}$, Zawar Hussain Khan ${ }^{2 a}$, Haseeb Khan ${ }^{2 b}$, Sheraz Khan ${ }^{1 b}$, \\ Suhail Khan ${ }^{3}$
}

RECEIVED ON 20.03.2019, ACCEPTED ON 26.07.2019

\begin{abstract}
Image denoising is a fundamental problem in both image processing and computer vision with numerous applications. It can be formulated as an inverse problem. Variational methods are commonly used to solve noise removal problems. The Total Variation (TV) regularization has evolved from an image denoising method for images corrupted with multiplicative noise into a more general technique for inverse problems such as denoising, deblurring, blind deconvolution, and inpainting, which also encompasses the Impulse, Poisson, Speckle, and mixed noise models. Multiplicative noise removal based on TV regularization has been widely researched in image science. In multiplicative noise problems, original image is multiplied by a noise rather than added to the original image. This article proposes a novel meshless collocation technique for the solution of a model having multiplicative noise. This technique includes TV and local collocation along with Multiquadric Radial Basis Function (MQ-RBF) for the solution of associated Euler-Lagrange equation for restoring multiplicative noise from digital images. Numerical examples demonstrate that the proposed algorithm is able to preserve small image details while the noise in the homogeneous regions is removed sufficiently. As a consequence, our method yields better denoised results than those of the current state of the art methods with respect to the Peak-Signal to Noise Ratio (PSNR) values.
\end{abstract}

Keywords: Image De-Noising, Multiplicative Noise, Speckle Noise, Total Variation, Multiquadric Radial Basis Functions, Partial Differential Equation, Mesh-Based Scheme, Meshless Scheme.

\section{INTRODUCTION}

I mage denoising is a research topic that has been studied by researchers from last many decades. Image noise removal problem is of two types, one is additive noise removal and the second one is the multiplicative noise removal problem. However multiplicative model noise removal is more challenging as compared to additive noise removal, therefore we focus on models for removing multiplicative noise, which can be stated as:

$\mathrm{g}=\mathrm{zn}$

where g: $\Omega \subset \mathrm{R}^{2} \rightarrow \mathrm{R}$ is the given noisy image, having multiplicative noise $\mathrm{n}$ and $\mathrm{z}$ is the original image.
Here, $\Omega$ represents a rectangular image domain. Multiplicative denoising is one of the most important and challenging task. The assignment of removing multiplicative noise has been proposed in many image processing fields, particularly, in medical sciences and Synthetic Aperture Radar (SAR) [1-4]. In the literature, various methods have been utilized for the numerical solution of Partial Differential Equations (PDEs) connected with models having multiplicative noise, for example, see [5-9].

The TV-based [10-12] methods have been proven one of the successful tools for the smooth solution of the associated Euler-Lagrange equation. The non-linearity and non-differentiability of TV-based model limits its

${ }^{1}$ Department of Electrical Engineering, University of Engineering and Technology, Mardan, Pakistan.

Email: ${ }^{a}$ mushtaq@uetmardan.edu.pk (Corresponding Author), ${ }^{\text {bsheraz@uetmardan.edu.pk }}$

${ }^{2}$ Department of Electrical Engineering, University of Engineering and Technology, Peshawer, Pakistan. Email: a ${ }^{2}$ zawarkhan@uetpeshawar.edu.pk, bhaseebkhan@uetpeshawar.edu.pk

${ }^{3}$ Department of Computer and Software Engineering, University of Engineering and Technology, Mardan, Pakistan. Email: suhail.khan@uetmardan.edu.pk

This is an open access article published by Mehran University of Engineering and Technology, Jamshoro under CC BY 4.0 International License. 
factorability in terms of computational complexity and processing time. To avoid the complexity in computation of exact solution, artificial time marching method is usually used for approximate computation of Euler-Lagrange equation. The problem with artificial time marching method is its slow processing due to its strict stability constraints in the time steps.

Here it can be concluded that the above mentioned methods struggle with the smooth solution. In this research study, we address meshless collocation scheme to minimize the above mentioned issues.

Recently, an increasing attention has been given to the development meshless Radial Basis Function (RBF) collocation methods for the numerical solution of PDEs. Majority of PDEs results have concerned steady state problems with smooth solutions. More recently, there has been a growing interest in applying meshless RBF collocation methods to time-dependent PDE problems, again to problems with sufficiently smooth solutions. The meshless RBF collocation methods have more points of interest and have exhibited superior accuracy as compared with traditional mesh-based numerical methods such as, Finite Difference Method (FDM) [13], Finite Element Method (FEM) [14], Finite Volume Method (FVM) [15-16], and pseudospectral method [17]. For further information [18-24].

The main idea behind the energy for of RBFs interpolation for multidimensional scattered data are investigated in [25]. In late decades, collocation techniques have been displayed to deal engineering and sciences issues. The PDE techniques based on the meshless strategies have superior and extremely helpful. In recent times meshless methods based on RBFs have observed more fruitful techniques for scattered data interpolation. RBF techniques are not fixed to a grid and hence come under the umbrella of class called meshless methods. These methods are smooth and conditionally positive definite [26-28]. The RBF approach is an important tool for defining smooth functions in important geometries due to meshless applications and spectral accuracy [29]. This paper proposes a collocation technique for image denoising which has not been reported in the existing literature to the best of our knowledge. In this work, we show the RBF mesh-less collocation technique
(Kansa strategy) for the nonlinear PDE arising in this model, where the RBF is used for the approximation of the solution of PDE. Kansa method is a domain type strategy, which has numerous features like the finite element approach for the approximation of the solution of PDE. For information about RBF strategies, see $[24,30,31]$.

The rest of the paper is organized as follows: Section 2, contains the details of RBFs and its applications in solving PDEs. Sections 3 discusses AA model [5]. Section 4 provides the detail discussion of numerical methods including mesh-based method and meshless method. Section 5 discusses and compares experimental results on various real and artificial images for image restoration. This section also includes to analyzes the shape parameter and sensitivity analysis of parameters for image restoration. Section 6 concludes the proposed work. Finally, the detail discussion for the derivative for the new meshless approach is given in an appendix.

\section{RADIAL BASIS FUNCTION}

Let us describe the RBF method now. The RBF is a function $\phi(\mathrm{x})$ with respect to the origin, $\phi(\mathrm{x})=$ $\phi(r) \in \mathrm{R}$, or on the distance a point from the given data set $\left\{x_{j}\right\}$ with $\phi\left(\mathrm{x}-\mathrm{x}_{\mathrm{j}}\right)=\phi\left(\mathrm{r}_{\mathrm{j}}\right) \in \mathrm{R}$ and $\phi(\mathrm{x})=$ $\phi(\|x\|)_{2}$ is known as radial function. Table1 shows some commonly used RBFs, such as Multiquadric (MQ), Inverse Multiquadric (IMQ), Genetic Algorithm (GA), and TPS. The RBF method is used to interpolate a smooth function $\mathrm{f}(\mathrm{x}), \mathrm{x} \in \Omega \subseteq \mathrm{R}^{\mathrm{n}}$, where $\Omega$ is the bounded domain. For given $\mathrm{N}$ interpolating values $\left\{y_{i}\right\}_{i=1}^{N} \in R$ and $\left\{x_{i}\right\}_{i=1}^{N} \in \Omega \subseteq R^{n}$ data centers, the $\mathrm{RBF}$ approximation is defined as

$\mathrm{f}(\mathrm{x})=\sum_{\mathrm{j}=1}^{\mathrm{N}} \gamma_{\mathrm{j}} \phi\left(\left\|\mathrm{x}-\mathrm{x}_{\mathrm{j}}\right\|_{2}\right), \quad \mathrm{x} \in \Omega$

where $\gamma_{j}$ are unknown weights. To find $\gamma_{j}$, the collocation RBF method is

$y_{i}=f\left(x_{i}\right)=\sum_{j=0}^{N} \gamma_{j} \phi\left(\left\|x-x_{j}\right\|_{2}\right), i, j=1,2, \cdots, N$ (3) which results in $\mathrm{N} \times \mathrm{N}$ linear system of equations which is given as follows.

$\mathrm{A} \alpha=\mathrm{B}$

where $\alpha=\left(\alpha_{1}, \alpha_{2}, \cdots, \alpha_{N}\right)^{t}$ is the $\mathrm{N} \times 1$ unknown vector and to be defined, $b=\left(\mathrm{y}_{1}, \mathrm{y}_{2}, \cdots, \mathrm{y}_{\mathrm{N}}\right)^{\mathrm{t}}$, is the 
$\mathrm{N} \times 1$ vector, and $\mathrm{A}=\left[\Phi_{\mathrm{ij}}\right]=\left[\phi\left(\left\|\mathrm{x}_{\mathrm{i}}-\mathrm{x}_{\mathrm{j}}\right\|_{2}\right)\right]_{1 \leq \mathrm{i}, \mathrm{j} \leq \mathrm{N}}$ with $\Phi_{\mathrm{ij}}=\Phi_{\mathrm{ji}}$, is called $\mathrm{N} \times \mathrm{N}$ interpolation matrix.

To ensure the invariability of the interpolation matrix A. he polynomial term is augmented to RBF equation.

In this case Equation (2) can be re-written as

$\mathrm{f}(\mathrm{x})=\sum_{\mathrm{j}=1}^{\mathrm{N}} \gamma_{\mathrm{j}} \phi\left(\left\|\mathrm{x}-\mathrm{x}_{\mathrm{j}}\right\|_{2}\right)+\sum_{\mathrm{i}=1}^{\mathrm{M}} \gamma_{\mathrm{N}+1} \mathrm{p}_{\mathrm{i}}(\mathrm{x})$

with constraints

$\sum_{\mathrm{i}=1}^{\mathrm{M}} \gamma_{\mathrm{j}} \mathrm{p}_{\mathrm{i}}\left(\mathrm{x}_{\mathrm{j}}\right), \quad \mathrm{i}=1,2, \cdots, \mathrm{M}$

With $\mathrm{p}_{\mathrm{i}} \in \prod_{\mathrm{m}-1}, \mathrm{i}=1,2, \cdots, \mathrm{M}$, where $\prod_{\mathrm{m}}$ shows the polynomial space in which the polynomials is $\mathrm{m}$ in $\mathrm{N}$ variables [26] are the total degree polynomials, which is given as:

$\left(\begin{array}{c}\mathrm{N}+\mathrm{m}-1 \\ \mathrm{~m}-1\end{array}\right)$

The interpolated solution of Equations (4-5) results in given matrix system $(\mathrm{M}+\mathrm{N}) \times(\mathrm{M}+\mathrm{N})$ equations.

$\left[\begin{array}{cc}\mathrm{A} & \mathrm{P} \\ \mathrm{P}^{\mathrm{t}} & 0\end{array}\right]\left[\begin{array}{l}\gamma \\ 0\end{array}\right]=\left[\begin{array}{l}\mathrm{b} \\ 0\end{array}\right]$,

in which $\quad A_{i, j}=\left[\Phi_{i j}\right]=\left[\phi\left(\left\|x_{i}-x_{j}\right\|_{2}\right)\right]_{1 \leq i, j \leq N}$ represents the elements of matrix $\mathrm{A}, \mathrm{P}_{\mathrm{i}, \mathrm{j}}=$ $\mathrm{p}_{\mathrm{i}}\left[\mathrm{x}_{\mathrm{j}}\right]_{1 \leq \mathrm{i} \leq \mathrm{N}, 1 \leq \mathrm{j} \leq \mathrm{M}}$ are the elements of matrix $\mathrm{P}$, and $\mathrm{O}$ is $\mathrm{M} \times \mathrm{M}$ matrix.

The RBFs with shape parameter $\mathrm{c}$, RBFs with positive definiteness (PD), and RBFs conditionality positive definiteness (CPD) are discussed in [27] and listed in

Table 1.

List of RBFs, where $m$ shows the m-order CPD [27], $[\mathrm{k}] \leq \mathrm{k}, \mathrm{N}$ is natural number. Shape parameter in RBF is represented by $\mathrm{c}$.

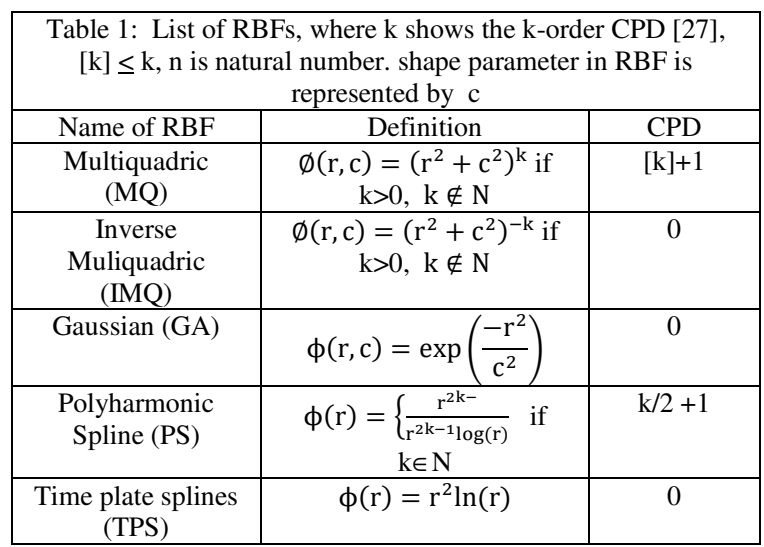

\section{AA MODEL}

Aubert et al. [5] proposed a non-convex Bayesian type of variational model for image denoising from given degraded image having multiplicative noise which includes the TV as the regularizer. The minimization functional of Equation (1) by [5] is formulated as:

$$
\begin{aligned}
& \inf \min _{\mathrm{z} \in \mathrm{S}(\Omega)} \mathrm{J}(\mathrm{z})+\lambda \int_{\Omega}\left(\log (\mathrm{z})+\frac{\mathrm{g}}{\mathrm{z}}\right) d x d y \\
& \text { where } \quad J(z)=\int_{\Omega} \nabla z \mid d x d y, \\
& S(\Omega)=\{z \in B V(\Omega), z>0\}
\end{aligned}
$$

In Equation (6) the first term is the TV regularization of $\mathrm{z}$ and $\lambda$ is the regularization parameter and the second term is called data fitting term respectively. The regularization parameter $\lambda$ is utilized to adjust the restoration and smoothness of the restored image which is usually depends on upon the noise level. Here, $g>0$ in $L_{\infty}(\Omega)$ shows the known information in the model. The minimization functional (6) then leads to the following Euler-Lagrange equation.

$-\nabla \cdot\left[\frac{\nabla \mathrm{z}}{|\nabla \mathrm{z}|^{2}+\beta}\right]+\lambda\left(\frac{\mathrm{z}-\mathrm{g}}{(\mathrm{z}+\beta)^{2}}\right)=0$ in $\Omega$,

or

$\frac{\partial}{\partial x}\left(\frac{z_{x}}{\sqrt{z_{x}^{2}+z_{y}^{2}}}\right)+\frac{\partial}{\partial y}\left(\frac{z_{y}}{\sqrt{z_{x}^{2}+z_{y}^{2}}}\right)+\lambda\left(\frac{z-g}{z^{2}}\right)=0$

The time dependent Euler-Lagrange Equation (8) is given as under.

$$
\frac{\partial z}{\partial t}=\frac{\partial}{\partial x}\left(\frac{z_{x}}{\sqrt{z_{x}^{2}+u_{y}^{2}}}\right)+\frac{\partial}{\partial y}\left(\frac{z_{y}}{\sqrt{z_{x}^{2}+z_{y}^{2}}}\right)+\lambda\left(\frac{z-g}{z^{2}}\right)
$$

for the given $z(x, y, 0)=\frac{1}{|\Omega|} \int_{\Omega} g$. For further information, the readers are refer to [5].

\section{NUMERICAL TECHNIQUES}

Now we present numerical schemes for solving nonlinear PDE Equation (9) associated with the minimization functional (6). 


\subsection{Traditional Mesh-Based Technique (M1)}

Aubert et. al used the mesh-based technique to solve the non-linear PDE (9). The PDE (9) is;

$\frac{\partial z}{\partial t}=\frac{\partial}{\partial x}\left(\frac{z_{x}}{\sqrt{z_{x}^{2}+z_{y}^{2}}}\right)+\frac{\partial}{\partial y}\left(\frac{z_{y}}{\sqrt{z_{x}^{2}+z_{y}^{2}}}\right)+\lambda\left(\frac{z-g}{z^{2}}\right)$

The explicit scheme is utilized to solve the above Equation (10) which is given as follow.

$\frac{z^{(n+1)}-z^{(n)}}{d t}=\frac{\partial}{\partial x}\left(\frac{z_{x}^{(n)}}{\sqrt{\left(z_{x}^{2}\right)^{(n)}+\left(z_{y}^{2}\right)^{(n)}}}\right)+$

$\frac{\partial}{\partial y}\left(\frac{z_{y}^{(n)}}{\sqrt{\left(z_{x}^{2}\right)^{(n)}+\left(z_{y}^{2}\right)^{(n)}}}\right)+\lambda\left(\frac{z^{(n)}-g^{(0)}}{\left(z^{2}\right)^{(n)}}\right)$

For further details, see in [5].

\subsection{Proposed Meshless Technique (M2)}

In this section, we present a new methodology by utilizing TV regularization alongside with RBFs to reestablish the clean image $\mathrm{z}$ from degraded image $g$ in the model given in Equation (1). Assume $\left\{\mathrm{x}_{\mathrm{i}}\right\}_{\mathrm{i}=1}^{\mathrm{N}}$ is the $\mathrm{N}$ distant evaluation points in $\Omega \subseteq R^{2}$ where $\Omega$ is a closed domain. Every RBF satisfy the following equation $\phi(r)=\|r\|_{2}$ in $R^{2}$ i.e $r=(x, y)$. For $\left\{x_{j}\right\}_{j=1}^{N c}$, given $\mathrm{Nc}$ centers, the RBF interpolation without polynomial term is defined as

$\mathrm{s}(\mathrm{x})=\sum_{\mathrm{j}=1}^{\mathrm{Nc}} \rho_{\mathrm{j}} \phi\left(\left\|\mathrm{x}-\mathrm{xc}_{\mathrm{j}}\right\|_{2}\right)$

coefficients of $\rho_{j}$ in above Equation (12) is resolved by means of upholding the interpolation condition $\mathrm{s}\left(\mathrm{x}_{\mathrm{j}}\right)=\mathrm{g}$

A collection of points that correspond to the centers $\mathrm{Nc}$. The RBF interpolation at $\mathrm{Nc}$ centers is given as $\mathrm{A} \rho=\mathrm{g}$

which gives a $\mathrm{Nc} \times \mathrm{Nc}$ linear system of equations and is solved for the coefficients $\rho$, where $\rho=$ $\left(\rho_{1}, \rho_{2}, \cdots, \rho_{\mathrm{Nc}}\right)^{\mathrm{t}}$ and $\mathrm{z}=\left(\mathrm{z}_{1}, \mathrm{z}_{2}, \cdots, \mathrm{z}_{\mathrm{Nc}}\right)^{\mathrm{t}}$ are $\mathrm{Nc} \times 1$ matrices. In the above equation $A$ is called the system or interpolation matrix and is defined as under.

$\mathrm{A}=\left[\Phi_{\mathrm{ij}}\right]=\left[\phi\left(\left\|\mathrm{xc}_{\mathrm{i}}-\mathrm{xc}_{\mathrm{j}}\right\|_{2}\right)\right]_{1 \leq \mathrm{i}, \mathrm{j} \leq \mathrm{Nc}}$
The matrix $\mathrm{A}$ in the above system is $\mathrm{Nc} \times \mathrm{Nc}$ square matrix which is invertible because it is always positive definite $[15,20]$. Thus

$\rho=\mathrm{A}^{-1} \mathrm{~g}$

with $\rho$ is a matrix of $\mathrm{Nc} \times 1$ order. In similar way, by using Equation (12) the RBF interpolation at $\mathrm{N}$ evaluation points $\left\{\mathrm{x}_{\mathrm{i}}\right\}_{\mathrm{i}=1}^{\mathrm{N}}$ results in $\mathrm{N} \times \mathrm{Nc}$ matrix $\mathrm{B}$ which is defined as follow.

$\mathrm{B}=\left[\Phi_{\mathrm{ij}}\right]=\left[\phi\left(\left\|\mathrm{x}_{\mathrm{i}}-\mathrm{xc}_{\mathrm{j}}\right\|_{2}\right)\right] \quad$ for $\quad \mathrm{i}=1,2, \ldots, \mathrm{N}$, $\mathrm{j}=1,2, . ., \mathrm{Nc}$

At $\mathrm{N}$ data points the interpolation condition is evaluated by using the matrix-vector product to produce $\mathrm{z}$ which is defined as under.

$z=\mathrm{B} \rho$

From Equations (13-14) the following equation is obtained.

$\mathrm{z}=\mathrm{BA}^{-1} \mathrm{~g}$

or

$z=\mathrm{Hg}$ where $\mathrm{H}=\mathrm{BA}^{-1}$

which results in the approximate solution of $\mathrm{N} \times 1$ matrix $\mathrm{z}$ at any point in $\Omega$. Since equation (11) is

Combining Equations (15-16) result in new restoration PDE, which is given as nonlinear system of equations.

$\mathrm{L}(\mathrm{z})^{(\mathrm{n})} \mathrm{z}^{(\mathrm{n}+1)}=\mathrm{L}(\mathrm{z})^{(\mathrm{n})} \mathrm{z}^{(\mathrm{n})}+\mathrm{dt}\left[\mathrm{z}_{\mathrm{xx}}^{(\mathrm{n})}+\mathrm{z}_{\mathrm{yy}}^{(\mathrm{n})}+\right.$

$\left.\mathrm{L}(\mathrm{z})^{\mathrm{n}} \lambda\left(\frac{\mathrm{z}^{(\mathrm{n})}-\mathrm{g}^{(0)}}{\left(\mathrm{z}^{2}\right)^{(\mathrm{n})}}\right)\right]$

where $L(z)=\sqrt{z_{x}^{2}+z_{y}^{2}}, \quad z_{x}=H_{x} g, \quad z_{y}=H_{y} g$,

$z_{x x}=H_{x x} g, z_{y y}=H_{y y} g$, and $g^{(0)}=g$.

As RBF collocation method is not required to satisfy the resultant PDE Equation (17), and have free choice to select RBF. The well-known RBF in collocation scheme is the MQ [25], which usually shows good results if suitable value for shape parameter is selected. The value of $c$ used in proposed meshless method M2 plays an important role for the smooth solution. For the best selected value of $\mathrm{c} \mathrm{M} 2$ results in good accurate and smooth outcome. The best experimental value of used in the proposed M2 results in smooth solution in image denoising. For the suitable value of c, M2 gives more accurate and smooth results in image denoising 
having multiplicative noise. In M2, c and $\lambda$ depend upon the size and noise in the tested image.

\section{Algorithm for Collocation Scheme M2: RBF:}

1. Select $\mathrm{N}=\mathrm{Nc}, \mathrm{n}$ number of points which are called pixel points.

2. Find the $\rho$ according to (13) by MQ-RBF.

3. Find $\mathrm{z}$ according to (15) by MQ-RBF.

Total Variation (TV) Filtering:

1. Select the initial values for $\lambda$, ò, $\mathrm{c}, \mathrm{dt}$ and $\mathrm{g}$.

2. Select $\mathrm{n}$ number of $\mathrm{Nc}$ points called centers i.e. $\mathrm{xc}_{1} \leq \mathrm{xc}_{2} \leq \cdots \mathrm{xc}_{\mathrm{n}}$, select $\mathrm{n}=0$.

3. Inset $\mathrm{z}$ as MQ-RBF in (17) from (15).

4. Select $n=n+1$. For the selection of each center point $\mathrm{xc}_{\mathrm{i}}$, for $\mathrm{i}=1,2,3, \ldots, \mathrm{n}$, calculate $\mathrm{Z}^{(\mathrm{n}+1)}$ from (17) by collocation method M2, where $\mathrm{g}^{(0)}=\mathrm{g}$.

5. $\frac{\left\|\mathrm{z}^{(\mathrm{n}+1)}-\mathrm{z}^{(\mathrm{n})}\right\|}{\left\|\mathrm{z}^{(\mathrm{n})}\right\|} \leq \grave{o}=10^{-3}$ (Stopping criteria), go to step (10).

6. Repeat step (7).

7. end.

8. Output $\mathrm{z}=\mathrm{z}^{(\mathrm{n}+1)}$

\section{EXPERIMENTAL RESULTS}

This section is dedicated to an examination of some numerically computed examples to show the execution of proposed strategy M2 over methods M1 on two types of multiplicative noises, to be specific multiplicative noise (Gamma distribution, mean 1 and variance $\mathrm{L}_{1}$ ) and speckle noise (Gamma distribution, mean 1 and variance $\mathrm{L}_{2}$ ). In order to get quantitative comparison, five original real and artificial images called, "Lena", "House", "SynImage1", "SynImage2" and "SynImage3" are used as test images which are appeared in Fig. 1(a-e).

In this work, it is supposed for proposed method M2 to select $\mathrm{N}=\mathrm{Nc}$, which denotes the size of the image for comparison with method M1. MQ-RBF is selected as basis function in M2. The indicator, PSNR is used to quantify the resultant denoised image. The PSNR is computed by the given equation.

$\operatorname{PSNR}=10 \times \log _{10}\left[\frac{\mathrm{m} \times \mathrm{nmax}\{\hat{\mathrm{z}}\}^{2}}{\|\hat{\mathrm{z}}-\mathrm{z}\|^{2}}\right]$

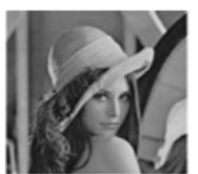

(a)

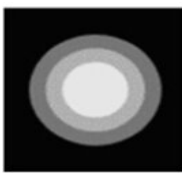

(d)

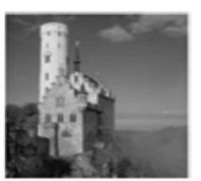

(b)

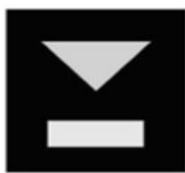

(e)

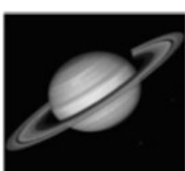

(c)
Fig. 1: Original Images for Our Experiments; (a) Lena; (b) House; (c) Synimage1; (d) Synimage2; (e) Synimage3.

where $\hat{\mathrm{z}}$ indicates the true image, $\mathrm{z}$ is the denoised image, and $m \times n$ represents the image size. The stopping criteria for iterations in the proposed scheme is given by the following condition.

$\frac{\left\|\mathrm{z}^{(\mathrm{n}+1)}-\mathrm{z}^{(\mathrm{n})}\right\|}{\left\|\mathrm{z}^{(\mathrm{n})}\right\|} \leq \grave{o}$

where òindicates the allowed permissible error and is selected as $10^{-3}$. At any point $\left(\mathrm{x}_{\mathrm{i}}, \mathrm{y}_{\mathrm{j}}\right)$, the formula for MQ-RBF is given as

$$
\begin{aligned}
& \emptyset_{j}(x, y)=\sqrt{c^{2}+r_{j}^{2}}=\sqrt{c^{2}+\left(\left(x-x_{j}\right)^{2}+\left(y-y_{j}\right)^{2}\right)} \\
& \text { where } r_{j}=\sqrt{\left(x-x_{j}\right)^{2}+\left(y-y_{j}\right)^{2}}
\end{aligned}
$$

Figs. 2-3 present the first experiment on real images "Lena" and "House". The original and noisy images with noise levels are shown in Fig. 2(a-b)-3(a-b), respectively. The denoised images by meshless approach M2 and mesh-based approach M1 are given in subfigures Figs. 2(c-d)-3(c-d), respectively. Subfigures Fig. 2(c-d)-3(c-d), respectively in this experiment for the two real images indicate that the image denoising performance of proposed colocation technique $\mathrm{M} 2$ is efficient than technique $\mathrm{M} 1$ due to the meshless applications of MQ-RBF applied in M2. The shape parameter $\mathrm{c}$ is an important parameter in image denoising in proposed meshless method M2 which affect the image restoration quality. In our case, the better choice of $c$ for two real images are set in1.71 $\leq$ $c \leq 1.75$. Furthermore, Table 2 indicates, the quality of image restoration for the two procedures M1 and M2. The denoising performance is measured by the greater value of PSNR. So the listed values of PSNR of M2 two real images "Lena" and "House" are larger than M1 which represents the successful restoration 
performance of M2 due to MQ-RBF applications over M1. Table 2, also shows the list of total computation time and iterative numbers required for convergence for algorithms M1 and M2. In Table 2, the total computation time and iterative numbers of $\mathrm{M} 2$ are smaller than M1 which indicates the fast restoration performance of M2 over M1 because of its meshless application. Hence, it can be concluded from the results given in Table 2 for real images "Lena" and "House" that the performance of proposed technique M2 is superior to technique M1.

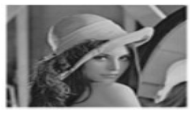

(a)

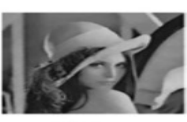

(d)

g. 2: Reconstructed results on Lena Image; (a) True Image; (b) Lena with multiplicative noise $\mathrm{I}_{1}=0.09$; (c) Obtained Result by Method M1 $(\lambda=443)$; (d) Obtained result by Method M2 ( $\lambda$ $=354, \mathrm{c}=1.74$ )

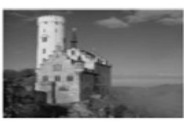

(a)

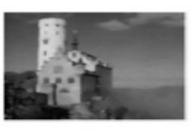

(d)

Fig. 3: Obtained Results on House Image; (a) True Image; (c) House Corrupted with Multiplicative Noise $L_{1}=0.09$; (c) Reconstructed Image Using M1 $(\lambda=437)$; (d) Reconstructed Image Using M2 $(\lambda=346, c=1.72)$

Figs. 4-5 show that the behavior of our technique M2 with complicated geometrical artificial images "SynImage1", "SynImage2". Again the original and noisy images with noise levels $\left(\mathrm{L}_{2}=0.01,0.01\right)$ are shown in subfigures Fig. 4(a-b)-5(a-b), respectively. This example shows that the result of image denoising

by collocation method M2 is better than mesh-based approach M1 due to the MQ-RBF applications used in M2. These resultant images can be seen from the subfigures Fig. 4(c-d) - 5(c-d), respectively. Since speckle noise is more complicated noise as compared to multiplicative noise. But still, the proposed methodology M2 produces good restoration results compared with the method M1. Again, the range for the best-selected values of $c_{\text {for }}$ the two tested images are set in $1.80 \leq c \leq 1.84$. The PSNR values, the total time of computation and iterative numbers selected for the tested images are listed in Table 2. Moreover, the PSNRs of the restored images by M2 is greater than M1, and also the total time of computation and iterative numbers of $\mathrm{M} 2$ are smaller than $\mathrm{M} 1$, which demonstrate the efficiency of collocation method M1 on mesh-based method M1. These results are listed in Table 2 .

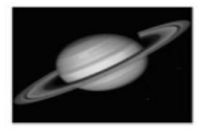

(a)

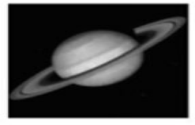

(d)

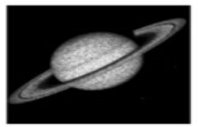

(b)

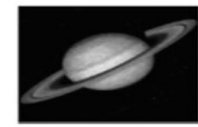

(c)

Fig. 4: Restoration Performance by two different methods on Synimage 1: (a) True Image (b) Noisy Image with Speckle Noise $L_{2}=0.1$ (c) Obtained Results by $M 1(\lambda=33)$; (d) Obtained Results by M2 $(\lambda=26, c=1.83)$.

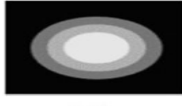

(a)

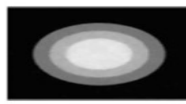

(d)

Fig 5: De-Noised Synimage2 Image with two different algorithms (a) True Image (b) Noisy Image $L_{2}=0.1$ (c) DeNoised Image By M1 $(\lambda=28)$ (d) De-Noised Result By M2 ( $\lambda$ $=23, c=1.81$ )

\begin{tabular}{l}
\hline \multicolumn{2}{|c|}{ Table 2: The PSNR values, computational time, and iterative numbers of M2 are compared with M1. The computational time is } \\
taken in seconds. \\
\hline \multirow{2}{*}{ Image }
\end{tabular}


In this paragraph, we discuss the homogeneity of the two algorithms M1 and M2 on an artificial image "SynImage1". We also check the preservation or loss of M1 and M2 on the same "SynImage1". For this purpose, a line of the true image is compared with the same lines of noisy and restored images that are given in Fig. 6. It can be seen from subfigure Fig. 6(c) that the line obtained by proposed meshless technique M2 is better than the line obtained by the technique M1 which is shown in subfigure Fig. 6(b).

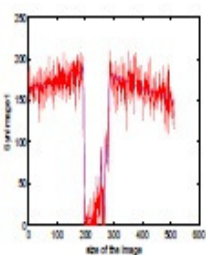

(a)

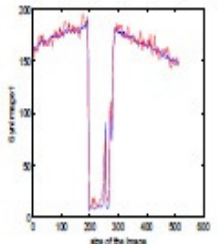

(b)

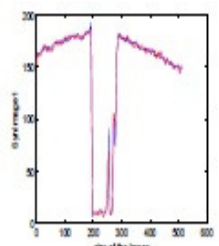

(c)
Fig. 6: The $110^{\text {th }}$ Line of True Image "Synimage1" Comparison with $110^{\text {th }}$ line of noisy image, restored by $\mathrm{M} 1$, and restored by M2 Images, respectively. (a) True and noisy images lines; (b) True and obtained images lines by $\mathrm{M} 1$; (C) True and obtained images lines by method M2. where the blue and The red lines show the true and restored images.

In this paragraph, we have tested real image "house" for three different sizes i.e., $256^{2}, 320^{2}$ and $400^{2}$ for the same multiplicative noise $\mathrm{L}_{1}=0.09$ to see the effect of shape parameters c and F. In Fig. 7, subfigures (a), (d), and (g), respectively show the noisy images of sizes $256^{2}, 320^{2}$ and with the same noise level $\mathrm{L}_{2}=$ 0.09. Subfigures (b), (e), (i) and (c), (f), (j) represent the restored images by methods M1 and M2, respectively. We can clearly see the image size effect the shape parameter $\mathrm{c}$ and $\mathrm{f}$ that are shown in in caption of Fig. 7. The performance of the two schemes M1 and M2 regarding image restoration (PSNR values) can be seen in Table 3 .

\begin{tabular}{|c|c|c|c|}
\hline \multicolumn{4}{|c|}{ Table 3: The Comparison of PSNR values for different } \\
sizes of House Image.
\end{tabular}

This section discusses the role of the shape parameter $\mathrm{c}$ regarding the image restoration used in the proposed collocation technique. We have applied and tested different values of c on "SynImage3" and noticed that these different values of $\mathrm{c}$ affect the image denoising quality (PSNR values), which are shown in Fig. 8 and Table 4.

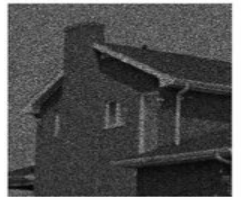

(a)

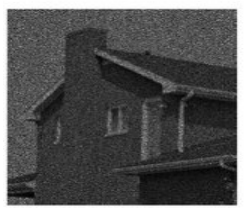

(d)

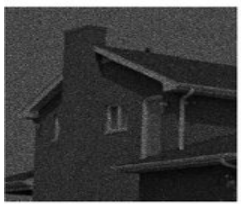

(g)

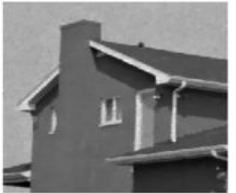

(b)

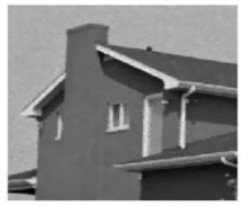

(e)

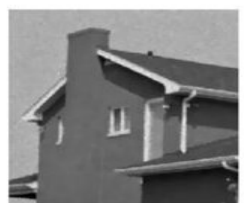

(h)

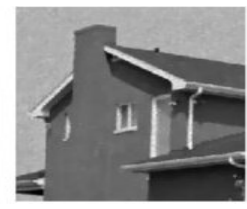

(c)

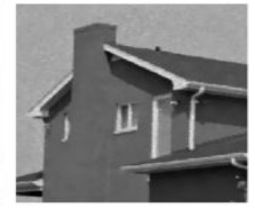

(f)

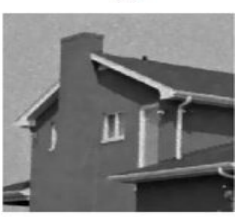

(i)
Fig. 7: Reconstructed Results nn House Image; (A) Noisy image with $L_{1}=0.09$ of size 256 ${ }^{2}$; (b) Obtained result by method M2 ( $\lambda=367, \mathrm{c}=1.71)$ (c) Obtained result By method M1 $(\lambda=482)$; (D) Noisy image with $L_{1}=0.09$ of size $320^{2}$; (e) Obtained result by Method M2 $(\lambda=350, c=1.75)$; $(\mathrm{f})$ Obtained result by Method M1 ( $\lambda=467) ;(g)$ Noisy image with $\mathrm{L}_{1}=0.09$ of size $400^{2} ;(\mathrm{H})$ Obtained result by Method M2 ( $\lambda$ $=343, \mathrm{C}=1.78)$; (I) Obtained result by method M1 $(\lambda=433)$.

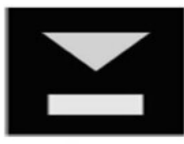

(a)

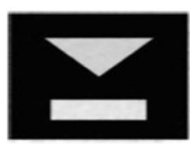

(d)

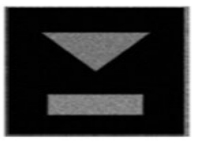

(b)

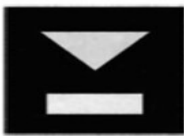

(e)
Fig. 8: Reconstructed image By $\mathrm{M} 2$ for artificial image Synimage3; (a) True Image (b) Degrade image with $L_{1}=$ 0.05 ; (c) Restored by optimal value $\mathrm{c}=180$; (d) Restored By $\mathrm{c}=1.87 ;$ (e) Restored By $\mathrm{c}=1.72$.

\subsection{Shape Parameter Analysis}

\begin{tabular}{|c|c|c|c|c|c|c|c|}
\hline \multicolumn{2}{|c|}{ Table 4: Image Restoration Quality (PSNR values) for various values of } & \multicolumn{1}{c|}{ Shape Parameter c M2 for artificial image } \\
\hline Image & Size & Optimal c & PSNR & Increase c & PSNR & Decrease c & PSNR \\
\hline Syn Image3 & $300^{2}$ & 1.80 & 25.30 & 1.87 & 25.09 & 1.72 & 24.96 \\
\hline
\end{tabular}




\subsection{Parameter Sensitivity Analysis}

The selection of parameters (c, $\lambda$ ) values used in the collocation method M2 is a difficult task according to our experience. Here, the image size and noise level are the two factors that are responsible for choosing the values of the parameters. The best experimental values of parameters are adjusted and tuned based on image size and noise variance.

It can be seen from the experimental results that the range of two parameters values used for different real and artificial images with different noise variance $\left(\mathrm{L}_{1}\right.$ $\left.=0.09,0.09, \mathrm{~L}_{2}=0.1,0.1\right)$ are $\mathrm{c} \in[1.65,1.85]$ and $\lambda \in[0.0000094,0.015]$. By selecting these ranges, better restoration results are achieved with improved PSNR results. The respective results are listed in Tables 5-6. In these Tables, $\uparrow$ and $\downarrow$ denote percent increase and percent decrease respectively.

\begin{tabular}{|c|c|c|c|c|c|c|}
\hline \multirow{2}{*}{ Image } & \multicolumn{3}{|c|}{$40 \%(\uparrow)$} & \multirow{2}{*}{\multicolumn{3}{|c|}{$70 \%(\uparrow)$}} \\
\hline & & & & & & \\
\hline Len & 2.43 & 495.6 & $2.31(\downarrow)$ & 2.96 & 6018 & 3390 \\
\hline
\end{tabular}

\begin{tabular}{|c|c|c|c|c|c|c|}
\hline \multicolumn{7}{|c|}{$\begin{array}{l}\text { Table 6: The Percentage effect on The PSNR values by } \\
\text { the Percentage decrease in parameters used in the } \\
\text { proposed scheme M2. }\end{array}$} \\
\hline \multirow{2}{*}{ Image } & \multicolumn{3}{|c|}{$40 \%(\downarrow)$} & \multicolumn{3}{|c|}{$70 \%(\downarrow)$} \\
\hline & $\mathrm{c}$ & $\lambda$ & PSNR & $\mathrm{c}$ & $\lambda$ & PSNR \\
\hline Lena & 1.04 & 212.4 & $\begin{array}{c}2.95 \\
(\downarrow)\end{array}$ & 0.52 & 106.2 & $\begin{array}{c}4.68 \\
(\downarrow)\end{array}$ \\
\hline
\end{tabular}

\section{CONCLUSION}

In this paper, a new meshless collocation technique that is MQ-RBF combined with TV regularization is proposed for multiplicative noise removal model. This approach is applied for the smooth solution of PDE associated with TV minimization functional. This meshless approach mathematically simple in used than the traditional method and hence obtain the fast and efficient restoration results.

This approach is examined with an exacting TV-based approach on various real and artificial images for multiplicative and speckle noises. Experimental results demonstrate that the performance of the proposed collocation technique is superior to the TVbased method regarding image restoration (PSNR values), total computational time, and iterative numbers. The shape parameter analysis and sensitivity analysis of parameters for better restoration performances have also been discussed.

\section{ACKNOWLEDGMENT}

The author acknowledges Prof. Dr. Siraj Ul Islam from Department of Basic Sciences, University of Engineering and Technology, Peshawar, Pakistan, for their administrative and technical support.

\section{REFERENCES}

[1] Achim, A., Bezerianos, A., and Tsakalides, P., "Novel Bayesian Multiscale Method for Speckle Removal in Medical Ultrasound Images", IEEE Transactions on Medical Imaging, Vol. 20, No 8, pp. 772-783, 2011.

[2] Bratsolis, E., and Sigelle, M., "Fast SAR image restoration, segmentation, and detection of high-reflectance regions", IEEE Transactions on Geoscience and Remote Sensing, Vol. 41, No 2, pp. 2890-2899, 2003.

[3] Malladi. R and Sethain, J, R., "A unified approach to noise removal, image enhancement, and shape recovery", IEEE Transactions on Image Processing, Vol. 5, No 11, pp. 1554-1568, 1996.

[4] Sheng, C., Xin, Y., Liping, Y., and Kun, S., "Total Variation-Based Speckle Reduction Using Multi-grid Algorithm for Ultrasound Images", International Conference on Image Analysis and Processing, Vol. 3617, pp.245252, 2005.

[5] Aubert, G., and Aujol, J, F., "A variational approach to removing multiplicative noise", SIAM Journal on Applied Mathematics, Vol. 68, No 4, pp. 925-946, 2008.

[6] Haung, L, L., Xia, L., and Huiwei, Z., "Multiplicative noise removal via a novel variation model", EURASIP Journal on Image and Video Processing, Vol. 5, pp. 987995, 2020.

[7] Le, T, M., and Vese, L, A., "Additive and Multiplicative Piecewise-Smooth Segmentation Models in a Variational Level Set Approach", Institute for Pure and Applied 
Mathematics, University of California at Los Angeles, Los Angeles, CA, Report 03-52, 2012

[8] Mei, Y., Ng, M, K., and Wen, Y, W., “A new total variation method for multiplicative noise removal", SIAM Journal on Imaging Sciences, Vol. 2, No 1, pp.20-40, 2009.

[9] Ogier, A., and Hellier, P., "A modified total variation denoising method in the context of 3D ultrasound images", International Conference on Medical Image Computing and Computer-Assisted Intervention, Vol. 3216, pp. 70-77, 2004.

[10] Rudin, L., Lions, P, L., and Osher, S., "Multiplicative denoising and deburring: theory and algorithms", Geometric Level Set Methods in Imaging, Vision, and Graphics, Vol. 3, pp. 103-119, 2003.

[11] Shi, J., and Osher, S., "A nonlinear inverse scale space method for a convex multiplicative noise model", SIAM Journal on Imaging Sciences, Vol. 1, No 3, pp. 294321, 2008.

[12] Rudin, L, L., Osher, S., and Fatemi, E., "Nonlinear total variation based noise removal algorithms", Physica D, Vol. 60, No 1-4, pp. 259-268, 1992.

[13] Zerroukat, M., Power, H., and Chen, C, S., "A numerical method for heat transfer problem using collocation and radial basis functions", International Journal for Numerical Methods in Engineering, Vol. 42, No 7, pp. 1263-1278, 1998.

[14] Li, J., Cheng, A., and Chen, C, S., "On the efficiency and exponential convergence of multiquadric collocation method compared with finite element method", Engineering Analysis with Boundary Elements, Vol. 27, No 3, pp. 251-257, 2003.

[15] Kansa, E, J., "Motivation for using radial basis functions to solve PDEs", Lawrence Livermore National Laboratory, USA, 1999.

[16] Li, J., and Hon, Y, C., "Domain decomposition for radial basis meshless methods", Numerical Methods for Partial Differential Equations, Vol. 20, No 3, pp. 450-462, 2004.
[17] Larsson, E., and Fornberg, B., “A numerical study of some radial basis function based solution methods for elliptic PDEs", Computers and Mathematics with Applications, Vol. 46, No 5-6, pp. 891-902, 2003.

[18] Kansa, E, J., and Holoborodko, P., "On the ill-conditioned nature of $\mathrm{C} \infty \mathrm{RBF}$ strong collocation", Engineering Analysis with Boundary Elements, Vol. 78, pp. 26-30, 2017.

[19] Fu, Z, J,. Chen, W., and Yang, H, T., "Boundary particle method for Laplace transformed time fractional diffusion equations", Journal of Computational Physics, Vol. 235, No 15, pp. 52-66, 2013.

[20] Fu, Z, J,. Xi, Q., Chen, W., and Cheng, A, H, D., "A boundary-type meshless solver for transient heat conduction analysis of slender functionally graded materials with exponential variations", Computers \& Mathematics with Applications, Vol. 76, No 4, pp. 760-773, 2018.

[21] Fu, Z, J., Yang, L, W., Zhu, H, W., and Hu, W, Z., " A semi-analytical collocation Trefftz scheme for solving multi-term time fractional diffusion-wave equations", Engineering Analysis with Boundary Elements, Vol. 98, pp. 137-146, 2019.

[22] Khowaja, S., and Shah, S, S., "Noise reduction technique for images using radial basis function neural networks", Mehran University Research Journal of Engineering \& Technology, Vol. 33, No 3, pp. 278-285, 2014.

[23] Javid, Q., Arif, M., Shah, M, A., and Nadeem, M., "A Hybrid Technique for DeNoising Multi-Modality Medical Images by Employing Cuckoo's Search with Curvelet Transform", Mehran University Research Journal of Engineering and Technology, Vol. 37, No 1, pp. 29-48, 2018.

[24] Chen, W., Fu, Z, J., and Chen, C, S., "Recent Advances in Radial Basis Function Collocation Methods", Briefs in Applied Sciences and Technology, Vol. 1, No. 23, 2013. 
[25] Kansa, E.J., "Multiquadrics - a scattered data approximation scheme with applications to computational fluid dynamics II: Surface approximations and partial derivative estimates", Computers and Mathematics with Applications, Vol. 19, No 8-9, pp. 127-145, 1990.

[26] Micchelli, C, A., "Interpolation of scattered data: Distance matrices and conditionally positive definite functions", Constructive Approximation, Vol. 2, No 1, pp. 11-12, 1986.

[27] Buhmann, M, D., "Radial Basis Functions: Theory and Implementations", Cambridge Monographs on Applied and Computational Mathematics, First Edition, 2004.

[28] Sarra, S, A., "Multiquadric Radial Basis Function Approximation Methods for the Numerical Solution of Partial Differential Equations", Article, Marshall University and Edward J. Kansa University of California, 2009.

[29] Driscoll, T, A., and Fornberg, B., "Interpolation in the limit of increasingly at radial basis functions.", Computers and Mathematics with Applications, Vol. 5, No 43, pp. 413-422, 2002.

[31] Haung, L, L., Xiao., L., and Huiwei, Z., "Multiplicative Noise Removal via a Novel Variational Model", EURASIP Journal on Image and Video Processing, Vol. 2010, No.1, pp.1-16, 2010.

[32] Sheng, C., Xin, Y., Liping, Y., and Kun, S., "Total Variation-Based Speckle Reduction Using Multi-grid Algorithm for Ultrasound Images", Proceedings of the International Conference on Image Analysis and Processing, Volume 13, pp. 245-252, 2005.

\section{APPENDIX}

The derivatives used in equation (17) in proposed algorithm $M_{2}$ are discussed below. For $\mathrm{N}$ evaluation points $\left\{\mathrm{x}_{\mathrm{i}}\right\}_{\mathrm{i}=1}^{\mathrm{N}}$ and $\mathrm{Nc}$ center points $\left\{\mathrm{x}_{\mathrm{j}}\right\}_{\mathrm{j}=1}^{\mathrm{N}}$ the RBF approximation is defend as under.

$\mathrm{z}=\sum_{\mathrm{j}=1}^{\mathrm{NC}} \rho_{\mathrm{j}} \phi\left(\left\|\mathrm{x}-\mathrm{xc}_{\mathrm{j}}\right\|_{2}\right)$ or $\mathrm{z}=\mathrm{B} \rho$ which results in $\mathrm{N} \times \mathrm{Nc}$ evaluation matrix B i.e., $B=\left[\Phi_{i j}\right]=\left[\phi\left(\left\|x_{i}-x_{j}\right\|_{2}\right)\right]$ for $i=1,2, \ldots N, j=$ $1,2, \ldots, \mathrm{Nc}$.

The first derivative from (20) is written as follow $\frac{\partial \mathrm{z}}{\partial \mathrm{x}_{\mathrm{i}}}=\mathrm{z}_{\mathrm{x}_{\mathrm{i}}}=\sum_{\mathrm{j}=1}^{\mathrm{Nc}} \rho_{\mathrm{j}} \frac{\partial}{\partial \mathrm{x}} \phi\left(\left\|\mathrm{x}-\mathrm{xc}_{\mathrm{j}}\right\|_{2}\right)$ or

$\mathrm{z}_{\mathrm{x}_{\mathrm{i}}}=\frac{\partial}{\partial \mathrm{x}_{\mathrm{i}}} \mathrm{B} \rho$,

where

$\frac{\partial \mathrm{B}}{\partial \mathrm{x}_{\mathrm{i}}}=\frac{\partial\left[\Phi_{\mathrm{ij}}\right]}{\partial \mathrm{x}_{\mathrm{i}}}=\frac{\partial}{\partial \mathrm{x}_{\mathrm{i}}}\left[\phi\left(\left\|\mathrm{x}_{\mathrm{i}}-\mathrm{xc}_{\mathrm{j}}\right\|_{2}\right)\right]$

for $\mathrm{i}=1,2, \ldots, \mathrm{N} \mathrm{j}=1,2, \ldots, \mathrm{Nc}$.

From equations (13) and (21) we get the given equation.

$\mathrm{z}_{\mathrm{x}_{\mathrm{i}}}=\frac{\partial}{\partial \mathrm{x}_{\mathrm{i}}} \mathrm{BA}^{-1} \mathrm{~g}$

Define $\mathrm{H}=\mathrm{BA}^{-1}$ the equation (22) is redefined as follow

$\mathrm{z}_{\mathrm{x}_{\mathrm{i}}}=\frac{\partial}{\partial \mathrm{x}_{\mathrm{i}}} \mathrm{H}_{\mathrm{x}_{\mathrm{i}}} \mathrm{g}$

The differentiation matrix is stated as

$\mathrm{H}_{\mathrm{x}_{\mathrm{i}}}=\frac{\partial}{\partial \mathrm{x}_{\mathrm{i}}} \mathrm{BA}^{-1}$

For second derivative, we have

$\mathrm{H}_{\mathrm{x}_{\mathrm{i}} \mathrm{x}_{\mathrm{i}}}=\frac{\partial^{2}}{\partial \mathrm{x}^{2}} \mathrm{BA}^{-1}$

Also

$\frac{\partial^{2} \mathrm{z}}{\partial \mathrm{x}^{2}}=\mathrm{z}_{\mathrm{x}_{\mathrm{i}} \mathrm{x}_{\mathrm{i}}}=\frac{\partial^{2}}{\partial \mathrm{x}_{\mathrm{i}}^{2}} \mathrm{Hg}=\mathrm{H}_{\mathrm{x}_{\mathrm{i}} \mathrm{x}_{\mathrm{j}}} \mathrm{g}$

As it is known that the system matrix $\mathrm{A}$ is invertible, so the differentiation matrix is well-defined.

For any sufficiently differentiable RBF, $[r(x)]$, the chain rule the first derivative is

$\frac{\partial \phi}{\partial x_{i}}=\frac{d \phi}{d r} \frac{\partial r}{\partial x_{i}}$ with $\frac{\partial r}{\partial x_{i}}=\frac{x_{i}}{r}$

The second derivative is defined as under.

$$
\begin{aligned}
& \frac{\partial^{2} \phi}{\partial \mathrm{x}_{\mathrm{i}}^{2}}=\frac{\mathrm{d} \phi}{\mathrm{dr}} \frac{\partial^{2} \mathrm{r}}{\partial \mathrm{x}_{\mathrm{i}}^{2}}+\frac{\mathrm{d}^{2} \phi}{\mathrm{dr} \mathrm{r}^{2}}\left(\frac{\partial \mathrm{r}}{\partial \mathrm{x}_{\mathrm{i}}}\right)^{2} \\
& \text { with } \frac{\partial^{2} \mathrm{r}}{\partial \mathrm{x}_{\mathrm{i}}^{2}}=\frac{1-\left[\frac{\partial \mathrm{r}}{\partial \mathrm{x}_{\mathrm{i}}}\right]^{2}}{\mathrm{r}}
\end{aligned}
$$

